\title{
Medical Image De-Noising Schemes Using Different Wavelet Threshold Techniques
}

\author{
Nadir Mustafa \\ School of Computer Science \& Technology, UESTC \\ Chengdu, 611731, China \\ Saeed Ahmed Khan \\ School of Microelectronics \& Solid Sate Electronics, \\ UESTC \\ Chengdu, 611731, China
}

\author{
Jiang Ping Li \\ School of Computer Science \& Technology, UESTC \\ Chengdu, 611731, China \\ Mohamed Tag Elsir \\ School of Electronic Engineering, UESTC \\ Chengdu, 611731, China
}

\begin{abstract}
In recent years most of researcher's has done tremendous work in the field of medical image applications such as Magnetic Resonance Imaging (MRI), Ultra Sound, CT scan but still there are many research and experiments in medical imaging field and diagnosing of human health by Health Care Institutes. There is a growing interest for medical imaging denoising as a hot area of research and also imaging equipment as a device. It is used for better image processing and highlighting the important features. These images are affected with random noise during acquisition, analyzing and Transmission process. This results in blurry image visible in low contrast. Wavelet transforms have effective method to separate the noise from the original medical image by using threshold techniques without affecting the important data of an image. Wavelet transform enables us to use the forward wavelet transform to represent subband of the original image in decomposition process then reconstructing this sub band coefficients to original image using inverse wavelet transform. In this work, the quality of medical image has been evaluated using filter assessment parameters like Variance, standard deviation, the squared difference error between original medical image $\&$ de-noised image (MSE) and the ratio between original image \& noisy image. From numerical results, we can see that the algorithm is efficient de-noising of noisy medical image. When, investigating with Baye's threshold techniques it achieved the Best value of peak signal to noise ratio (PSNR). For best medical image de-noising, the wavelet based denoising algorithm has been investigated and results of Baye's techniques and hard \& soft threshold methods have been compared.
\end{abstract}

Keywords-Baye's Wavelet threshold; Discrete Wavelet; Medical Image De-noising; Magnetic Resonance Imaging (MRI)

\section{INTRODUCTION}

Most of medical diagnostic equipment has applications such as magnetic resonance imaging (MRI), criminal identification systems (CIS), agricultural and biological research (ABR) uses the concept of digital image processing. The term image de-noising is the best tool used in these applications, where it effectively captures the noise from corrupted medical image and preserving with the valuable data and important features of the medical image [1][2].

The motivation of using Medical Resonance Imaging (MRI) as a hot area because of it's related with human health.
The medical Resonance imaging (MRI) very useful and low cost in diagnosis the human health and mapping the diagnosis output of the medical image in real and refine it as image quality [3][4].

During image acquisition and transmission, it has been usually observed that random noise always occurs at another end. In previous work, many researchers achieved good results in PSNR but not in MSE or visa-versa. Our work gives Good results in both PSNR and MSE [6][7].

Most of medical images are vulnerable to noise. This noise causes problems such as a blurred vision of images. Therefore, it is not easy for the medical doctors to examine the abnormalities in human in the invisible image. Most of medical imaging applications have been affected with random noises during Acquisition and transmission process that required improve and recover hidden data and details coefficients from noisy medical image.

Baye's threshold techniques provide good results when compared to Soft and Hard thresholds in terms of MSE and PSNR values as shown in the simulation results.

\section{WAVELET TRANSFORM}

The main difference between wavelet transform and windowed Fourier transform lies in signal analysis, the wavelet transforms using decomposition process to localize the signal in real time domain and frequency domain. In contrast windowed Fourier analysis has ability to localize the signal in Fourier space domain. Both Fourier and wavelet analysis represent the signal in different version such as sine wave and shifted and scale version which is kind of mother wavelet. But they are similar in windowing scales. The sine wave Fourier transforms have unlimited duration compared with waveform of wavelet transform and the wavelet forward to be irregular waveform. The wavelet Mathematical formula can be written as follows [5]. The equation (1) has been expressing a mathematic syntax of Fourier analysis and transform:

$$
F(\omega)=\int_{-\infty}^{\infty} f(t) e^{-j \omega t} d t
$$


Which mathematically is the sum over all time of the signal $\mathrm{f}(\mathrm{t})$ multiplied by Exponential formula. (In mathematic the Exponential formula can be expressed as real and imaginary of sinusoidal vectors).

The Fourier coefficients F $(\omega)$ and sinusoid components of original image are results of applying Fourier transform in image processing, when multiplied by sinusoid of frequency. Similarly, the equation (2) expressing mathematic syntax of continuous wavelet transforms which is integration over whole time of the signal multiplied by shift and scale version of the wavelet function.

$C($ scale, position $)=\int_{-\infty}^{\infty} f(t) \psi($ scale, position,$t) d t$

Many wavelet coefficients $\mathrm{C}$ are generated as function of scale and position, while Appling CWT in image processing by multiplied each coefficient with CWT function which consists of scale and shift as wavelet parameter [10].

In 1976 Croiser, Esteban, and Galand proposed discrete wavelet transform (DWT) as an effective technique to decompose the discrete time signals. On the other hand, Crochiere, Weber, Flanagan proposes the coding of speech signals technique which achieved as same time. The discrete wavelet transform DWT is sample of wavelet series and CWT version, which support sufficient and frequent information for wavelet decomposition and reconstruction of the original image, DWT requires sufficient amount of computations and resources. Furthermore, the DWT have ability to computerize the continuous wavelet transform as discretized version of signals.

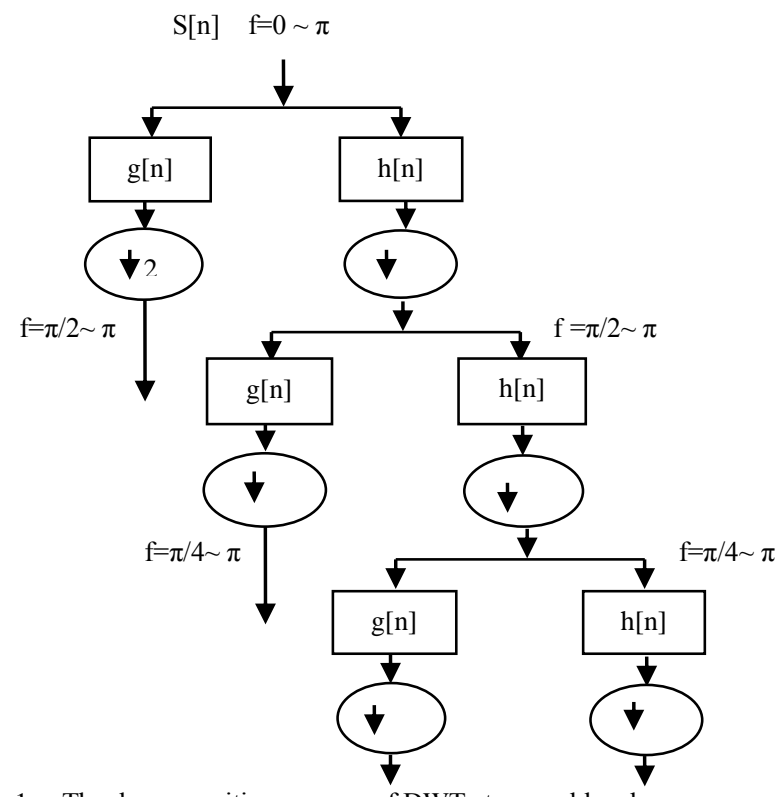

Fig. 1. The decomposition process of DWT at second level

Fig. (1) Depicts the decomposition of the signals by using high pass and low pass filters respectively to analyze the low and high frequencies and then measuring the amount of detailed information in the signal by using up sampling and down sampling operation. Both up sampling and down sampling terms respectively defined as reduction or remove the sample of signal, on other hand it means increase or adding new sample to the signal using Quadratic Mirror Filters which consist of two filters high pass and low pass. The DWT decompose the signal $\mathrm{S}[\mathrm{n}]$ simultaneously by passing through to the high pass filter $\mathrm{h}[\mathrm{n}]$ and low pass filter $\mathrm{g}[\mathrm{n}]$, which generate the details coefficients and approximation coefficients that respectively named [HH, HL, LH] and [LL]. While using the Quadratic Mirror Filters the half of frequencies signal are removed [9][11]. The following figure is describing the decomposition of DWT at second level.

\section{THRESHOLDING}

The threshold techniques is an effective and necessary tools in wavelet transform which used to calculate the wavelet coefficients using 1-D or 2-D or 3-D dimensional wavelet array $A[i, j]$ sized with $i, j=1$ to $M$ that is define the element numbers of array. The $\mathrm{Tb}$ is a threshold parameter which remains the wavelet coefficient and wavelet power in thresholding process. The scientists Donoho and Johnstone gave the best choice of threshold in (1994). They showed that the threshold discards the smaller wavelet coefficients and preserve the larger coefficients than threshold level [12] [13].

\section{A. Hard threshold}

The hard threshold techniques deal with wavelet coefficients that less than threshold level after computed the wavelet transform and inverse wavelet transform by sitting all the coefficients to zero. Mathematically, the hard threshold formula is represented as follow in equation (3).

$$
w_{t}=\left\{\begin{array}{c}
w_{t}\left|w_{t}\right|>T_{b} \\
0\left|w_{t}\right| \leq T_{b}
\end{array}\right.
$$

\section{B. Soft threshold}

The soft threshold work similar to hard threshold discarded all the coefficients less than threshold level to zero. It's also minimizing the magnitude of preserve wavelet coefficients to be equal with largest discarded coefficient. The soft threshold formula is represented as follow in equation (4)

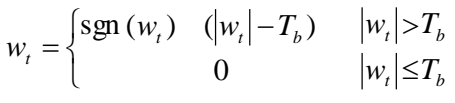

\section{Proposed De-Noising AlgorithM}

The Baye's threshold technique has a ability to forming the threshold wavelet sub band coefficients from medical image in Bayesian Frame Work (BFW) during the decomposition process for wavelet coefficients, In Fig. 2, De-nosing algorithm model using discreet wavelet transform (DWT) at 2nd- level of decomposition have been proposed. It has been assumed in Generalized Gaussian distribution (GGD) and carefully finds the threshold $\mathrm{Tb}$ to reduce the thresholding risk, which affect to the important data in wavelet details and approximation coefficients. Finally, the performance of this thresholding is better for de-noising medical image compared with other threshold techniques [14][16]. 


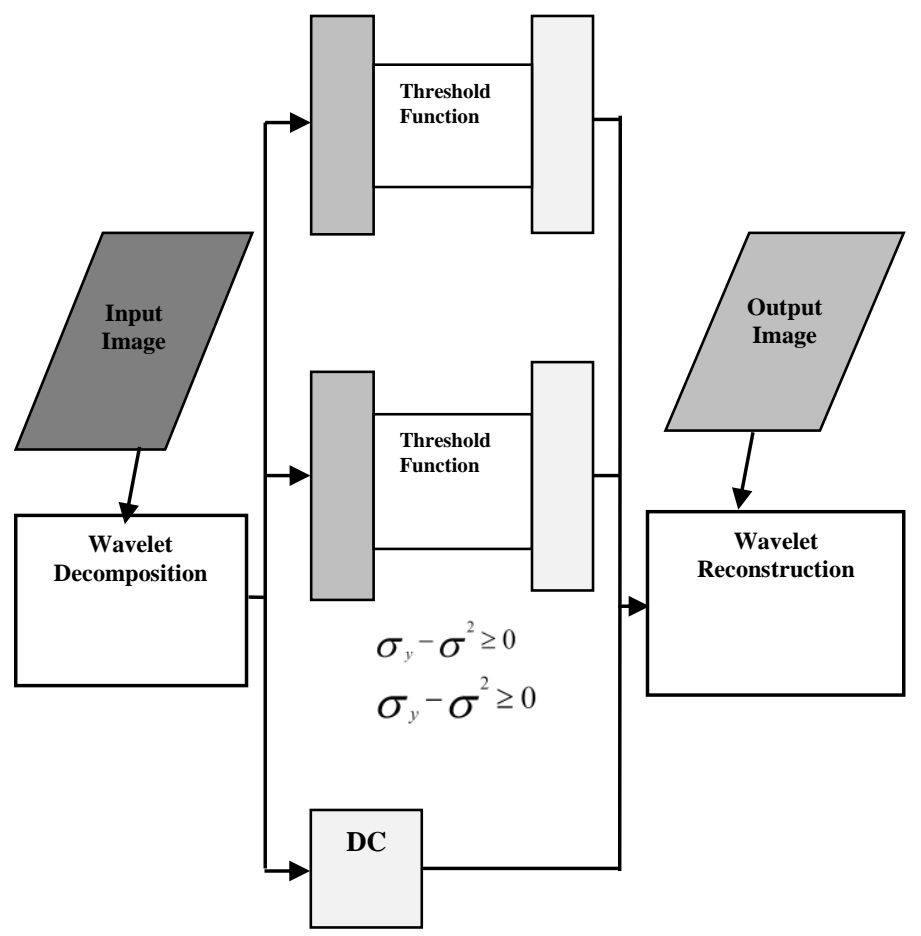

Fig. 2. Proposed De-nosing Algorithm Model using discreet wavelet transform (DWT) at 2nd- level of decomposition

\section{A. Estimation Parameters for Proposed Algorithm}

This section defines the adaptive parameters to proposed de-noising algorithm for medical image decomposed sub band coefficients which determine the threshold level $\left(T_{b}\right)$ in equation (5) for different details and approximation sub band coefficients that depend on sub branch equation, which compare the max value of difference variances compared to zero $\sigma_{\mathrm{y}}-\sigma 2 \geq 0$, by equation ( 3 ).

$$
\begin{aligned}
& T_{b}=\sigma^{2} / \sigma_{x} \\
& T_{b}=\max (a b s(X))
\end{aligned}
$$

Apply the wavelet transforms to medical image you have to generate details coefficient and approximation coefficients. From sub-band $\mathrm{HH}$ the noise variance measurement $\sigma^{2}$ is computed by the median estimator shown in equation (7).

$\sigma^{2}=\left[\frac{X-\text { median }\left(\left|Y_{i j}\right|\right)}{0.6745}\right]^{2}, Y_{i j} \in$ subband

The details coefficients and sub band [LH, HL and $\mathrm{HH}$ ] are generated while using wavelet decomposition process, the signal variance measurement $\sigma_{x}$ estimated by equation (8) using standard MATLAB command.

$$
\sigma_{x}=\sqrt{\left(\max \left(\sigma_{y}-\sigma^{2}, 0\right)\right)}
$$

And $\sigma \mathrm{x}$ is the signal variance without noise defined as the squired max value of differences variance of (noise image and additive white noise image) which compared to zero, performed using standard MATLAB command.

$\sigma_{y}=\frac{\sum(X)^{2}}{l_{k}}$
The standard deviation is defined as the average amount by which individual data from medical image sub band confidents differ from the arithmetic mean of all the data in the set. Lk is the length of the sub band at kth scale.

\section{IMAGE De-Noising AlgORITHM}

The Image De-noising Algorithm is used to extract the noise from during acquisition and transmission and achieve the best image quality in medical image processing, after performed the discrete wavelet transform for original image, and then implementing the bayes wavelet threshold to remove the noise, which affected the visibility of the medical image. And finally the inverse wavelet transform performed to recover the clarity of decomposed medical image. As following steps:

\section{A. Perform the decomposition on the two of original medical images $X$, $Y$ corrupted by Additive Gaussian Noise using wavelet transform}

B. Chose the bayes threshold from equation (5) or (6) by estimate the measurements

a) Estimate the noise variance $\sigma 2$ using equation (7).

b) Estimate the additive white noise variance $\sigma y$ using equation (9).

c) Estimate the signal variance $\sigma \times x$ using equation (8).

C. Perform the Reconstruction on the two of de-noised medical image $X^{\wedge}, Y^{\wedge}$ using inverse wavelet transforms to fine the clear vision of the image.

\section{EXPERIMENTS ANALYSIS AND OUTPUTS}

The laboratory work implemented on the two of medical images with same size $(256 \times 256)$ named (gland pituitary \& Prostate). These images are defined as Xs, r \& Ys, r matrix, which taken $\mathrm{s}, \mathrm{r}=1$ to $\mathrm{N}$ parameter as image size, and it is a gray scale image. The problems were occurred while transmission and acquisition of original image. The image was affected by noise which could not be diagnosed by doctors and health institutes due to invisibility in image while investigating patient. To overcome on this issue, the forward wavelet transforms, bayes wavelet threshold and inverse wavelet transform techniques are effective tools to separate the noise from original images using wavelet decomposition \& reconstructions process. After decomposition, bayes threshold have been applied for wavelet details coefficients and to assess the performance, the de-noising threshold algorithm have to add the variance noise measurement $\sigma 2=0.04$ of white Gaussian noise to original image for getting high peak signal to noise ratio (PSNR) measurement an d low mean square error (MSE) to assess the quality of reconstructed image compared with wavelet soft and hard threshold that measured by the equation (10)

$$
P S N R=10 \log _{10} \frac{255^{2}}{m s e}
$$

Where the signal to noise ratio measurement is detıned as the ratio of signal power to the de noise power of the denoised medical image, often expressed in decibels 
$M S E=\frac{s}{s \times r} \sum_{s=1}^{N} \sum_{r=1}^{N}\left[X(s, r)-X^{\wedge}(s, r)\right]^{2}$

The mean square error (MSE) of medical image in equation (11) is an estimator, which measures the average of

the square "errors", that is, the difference error between original medical images $(\mathrm{X})$ and synthesis image $\left(\mathrm{X}^{\wedge}\right)$. The Experimental work have been carried out by using bayes threshold to estimate the medical image quality using PSNR \& MSE measurements with various wavelet threshold methods and various wavelet packages which have been presented in table I, II. From the tables, it can be understand that the lower value of MSE and higher value of PSNR in bayes threshold is vice versa in soft threshold and hard threshold. Which reveals that, bayes threshold in medical image de-noising is effective tool based on the experimental results. The bayes threshold presents the visual quality while compared with soft and hard. Moreover, Bayes threshold has high efficiency and good performance than soft and hard threshold. The MSE ratio of Soft threshold, hard threshold and Bayes threshold of gland pituitary and prostate image have been shown in figure (3\&4). While figure (5\&6) depicts the PSNR ratio of Soft threshold, hard threshold and Bayes threshold of gland pituitary and prostate image.

TABLE I. MSE Result of Gland Pituitary \& Prostate Images Test WITH VARIENCE 0.04

\begin{tabular}{|l|l|c|c|c|}
\hline $\begin{array}{l}\text { Medical } \\
\text { Images }\end{array}$ & $\begin{array}{l}\text { Wavelet } \\
\text { Package }\end{array}$ & $\begin{array}{l}\text { Soft } \\
\text { Threshold }\end{array}$ & $\begin{array}{l}\text { Hard } \\
\text { Threshold }\end{array}$ & $\begin{array}{l}\text { Bayes } \\
\text { Threshold }\end{array}$ \\
\hline \multirow{4}{*}{$\begin{array}{l}\text { gland } \\
\text { pituitary } \\
\text { Image }\end{array}$} & haar & 0.0587 & 0.0570 & 0.0548 \\
\cline { 2 - 5 } & sym4 & 0.0570 & 0.0556 & 0.0537 \\
\cline { 2 - 5 } & $\mathrm{db} 4$ & 0.0559 & 0.0548 & 0.0530 \\
\cline { 2 - 5 } & bior3.7 & 0.0541 & 0.0534 & 0.0524 \\
\hline \multirow{4}{*}{$\begin{array}{l}\text { Prostate } \\
\text { Image }\end{array}$} & haar & 0.0652 & 0.0620 & 0.0601 \\
\cline { 2 - 5 } & sym4 & 0.0645 & 0.0616 & 0.0593 \\
\cline { 2 - 5 } & db4 & 0.0626 & 0.0594 & 0.0575 \\
\cline { 2 - 5 } & bior3.7 & 0.0595 & 0.0577 & 0.0566 \\
\hline
\end{tabular}

TABLE II. PSNR Result of Gland Pituitary \& PRostate Images TEST WITH VARIENCE 0.04

\begin{tabular}{|l|l|l|l|l|}
\hline \multirow{2}{*}{$\begin{array}{l}\text { Medical } \\
\text { Image }\end{array}$} & $\begin{array}{l}\text { Wavelet } \\
\text { Package }\end{array}$ & $\begin{array}{l}\text { Soft } \\
\text { Threshold }\end{array}$ & $\begin{array}{l}\text { Hard } \\
\text { Threshold }\end{array}$ & $\begin{array}{l}\text { Bayes } \\
\text { Threshold }\end{array}$ \\
\hline \multirow{4}{*}{$\begin{array}{l}\text { gland } \\
\text { pituitary } \\
\text { Image }\end{array}$} & haar & 32.1694 & 32.2001 & 32.2284 \\
\cline { 2 - 5 } & sym4 & 32.1575 & 32.1703 & 32.2034 \\
\cline { 2 - 5 } & $\mathrm{db} 4$ & 32.1404 & 32.1689 & 32.1921 \\
\cline { 2 - 5 } & bior3.7 & 32.1204 & 32.1404 & 32.1704 \\
\hline \multirow{3}{*}{$\begin{array}{l}\text { Prostate } \\
\text { Image }\end{array}$} & haar & 31.1594 & 31.1671 & 31.1904 \\
\cline { 2 - 5 } & sym4 & 31.1575 & 31.1703 & 31.1834 \\
\cline { 2 - 5 } & db4 & 31.1454 & 31.1589 & 31.1661 \\
\cline { 2 - 5 } & bior3.7 & 31.1342 & 31.1404 & 31.1584 \\
\hline
\end{tabular}

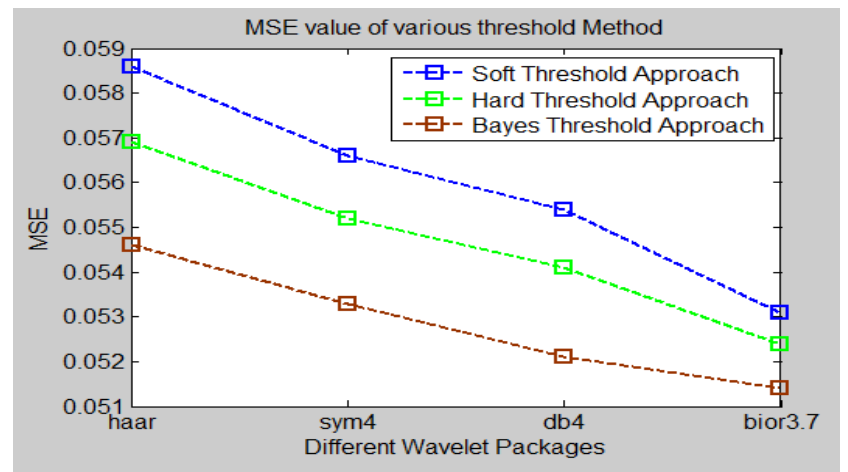

Fig. 3. Comparison of MSE of gland pituitary image de- noising; using different threshold techniques

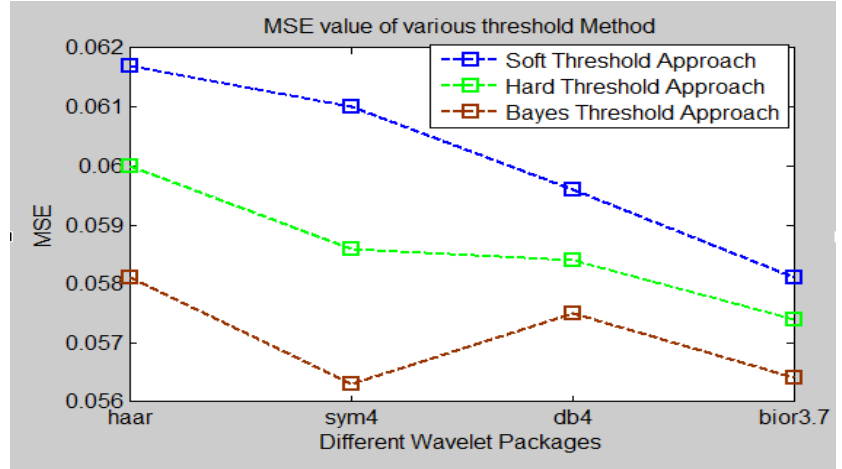

Fig. 4. Comparison of MSE of Prostate image de- noising; using different threshold techniques

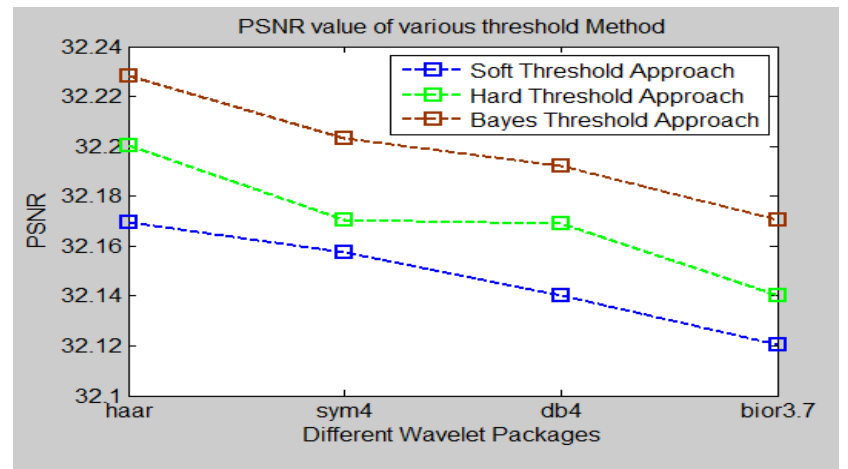

Fig. 5. Comparison of PSNR of Gland Pituitary image de- noising; using different threshold techniques

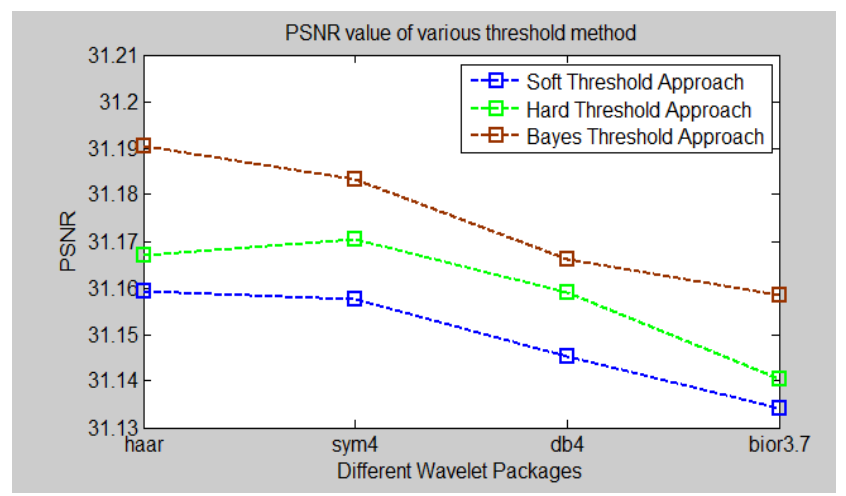

Fig. 6. Comparison of MSE of Prostate image de- noising; using different threshold techniques 
The Comparison analysis of soft, hard and bayes threshold can be visualized from figure $7 \& 8$. It can be observed that, the soft and hard threshold in gland pituitary and prostate images are blurred than the bayes threshold. It reveals that, according to the experimental results, bayes threshold technique has much worth over soft and hard threshold for medical image de-nosing.

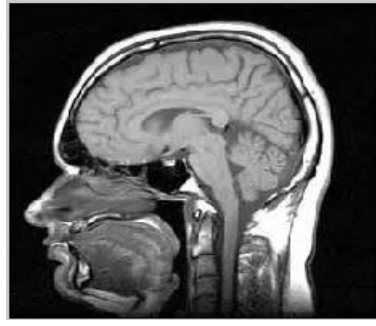

(a)

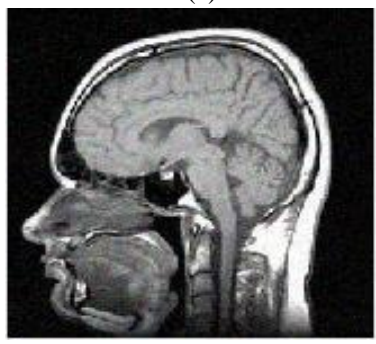

(c)

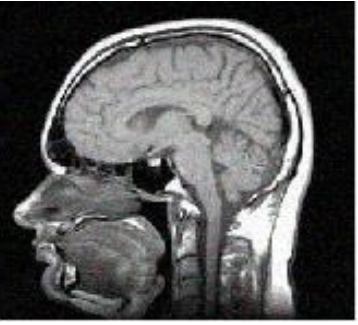

(b)

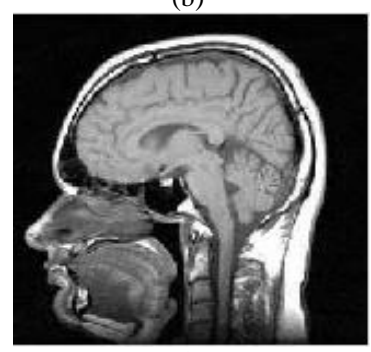

(d)

Fig. 7. Comparing the performance of gland pituitary image de- nosing (a) Original image (b) Soft threshold (c) Hard threshold (d) Bayes threshold

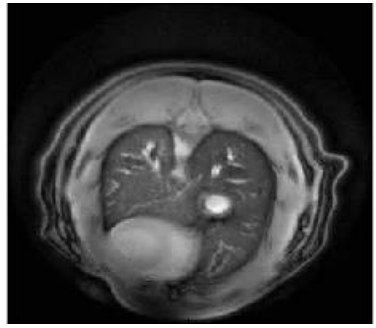

(a)

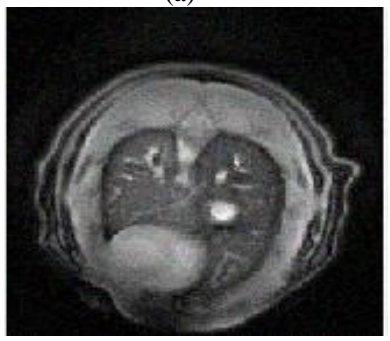

(c)

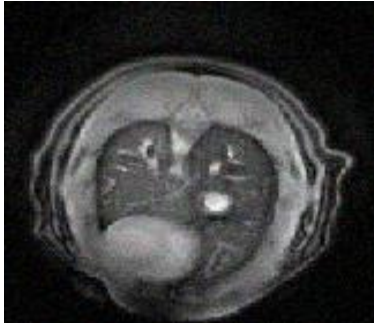

(b)

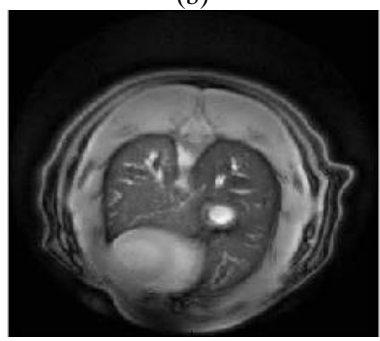

(d)
Fig. 8. Comparing the performance of prostate image de- nosing (a) Original Image (b) Soft threshold (c) Hard threshold (d) Bayes threshold

\section{CONCLUSION}

Nowadays, Magnetic Resonance Imaging (MRI) plays an important role in medical image processing applications. In this work Bayes wavelet methods provide powerful tools for data de-noising problems. As we have applied various method on brain \& prostate image using different wavelet threshold techniques (Soft, Hard and Baye's) and different wavelet packages ( Haar, Sym4, db4 and bior3.7) and among these threshold techniques, the Baye's threshold has an optimal performance compared to the soft \& hard threshold. In this method PSNR has maximum value \& MSE lower value and the vision of medical image is much clear as compared to the soft and hard thresholds. Bayes threshold technique removed the noise significantly and remains the important features of data and medical image.

For future work, I suggest using different kinds of images. Like CIS, Agricultural, biological and geographical images for mapping and navigation.

\section{ACKNOWLEDGMENT}

This paper was supported by National Natural Science Foundation of China (grant NO: 61370073), the national high technology research and development program of china (Grant No: 2007AA01z423)

\section{REFERENCES}

[1] Maarten Jansen. Noise Reduction by Wavelet Thresholding, volume 161. Springer Verlag, United States of America, 1 edition, 2001.

[2] Martin Vetterli S Grace Chang, Bin Yu. Adaptive wavelet Thresholding for image de-noising and compression. IEEE Transactions on Image Processing, 9(9):1532-1546, Sep 2000.

[3] Iain M.Johnstone David L Donoho. Adapting to smoothness via wavelet shrinkage. Journal of the Statistical Association, 90(432):1200-1224, Dec 1995.

[4] David L Donoho. Ideal spatial adaptation by wavelet shrinkage. Biometrika,

[5] David L Donoho. De-noising by soft Thresholding. IEEE

[6] S. G. Chang, B. Yu, M. Vetterli: IEEE Trans. Image Processing, (2000) 9 p. 1532

[7] S. G. Chang, B. Yu, M. Vetterli: IEEE Trans. Image

[8] D.L. Donoho, De-Noising by Soft Thresholding, IEEE Trans. Info. Theory 43, pp. 933-936, 1993.

[9] Javier Portilla, Vasily Strela, Martin J. Wainwright, Eero P. Simoncelli, Adaptive Wiener Denoising using a Gaussian Scale Mixture Model in the wavelet Domain, Proceedings of the 8th International Conference of Image Processing Thessaloniki, Greece. October 2001.

[10] Michel Misiti, Georges Oppenheim, and Jean-Michel Poggi, Wavlet Toolbox User's Guide, $\square \square$ COPYRIGHT 1996 - 1997 by The Math Works, Inc. All Rights Reserved.

[11] Robi Ploikar, Index to series of tutorials to wavelet tranfrom ,The Wavelet Tutorial was originally developed and hosted (1994-2000)

[12] S. Grace Chang, Bin Yu and M. Vattereli, Adaptive Wavelet Thresholding for Image Denoising and Compression, IEEE Trans. Image Processing, vol. 9, pp. 1532-1546, Sept. 2000.

[13] D.L. Donoho and I.M. Johnstone, Adapting to unknown smoothness via wavelet shrinkage, Journal of American Statistical Assoc., Vol. 90, no. 432, pp 1200-1224, Dec. 1995.

[14] S. Grace Chang, Bin Yu and M. Vattereli, Wavelet Thresholding for Multiple Noisy Image Copies, IEEE Trans. Image Processing, vol. 9, pp.1631- 1635, Sept.2000.

[15] S. Grace Chang, Bin Yu and M. Vattereli, Spatially Adaptive Wavelet Thresholding with Context Modeling for Image Denoising,, IEEE Trans. Image Processing, vol. 9, pp. 1522-1530, Sept. 2000.

[16] M. Vattereli and J. Kovacevic, Wavelets and Subband Coding. Englewood Cliffs, NJ, Prentice Hall, 1995

[17] Pooja Kaushik and Yuvraj Sharma,Comparison Of Different Image Enhancement Techniques Based Upon Psnr \& Mse,International Journal of Applied Engineering Research, ISSN 0973-4562 Vol.7 No.11 (2012)

[18] Lakhwinder Kaur, Savita Gupta and R.C. Chauhan, Image Denoising using Wavelet Thresholding, 